\title{
Mathematical and empirical description of screen blocking
}

\author{
Lawinska Katarzyna $^{1,2} \cdot$ Modrzewski Remigiusz $^{1} \cdot$ Wodzinski Piotr $^{1}$
}

Received: 29 January 2015 / Published online: 26 February 2016

(C) The Author(s) 2016. This article is published with open access at Springerlink.com

\begin{abstract}
This paper addresses the process of sieve holes blocking taking place while screening fine-particulate materials and provides an analytical description of the phenomenon. Empirical tests performed as part of this research provided dependences specifying the parameters of sieve holes blocking that could be applied in practice. The process of sieve holes blocking is unfavourable and inevitable. It is related with the screen clearance coefficient which is one of the fundamental characteristics of all screens. The grains whose dimensions are similar to the dimensions of the sieve holes block those holes, causing their exclusion from the active surface area of the screen. This phenomenon results in diminished performance and efficiency of the screening process. Screening is a very common industrial practice, and various designs of screens and types of sieves are available. That is why the subject of this paper is so important. The screen blocking coefficient is the measure of sieve holes blocking. In order to describe the process of screen blocking, a mathematical model is presented and a method for determining the screen blocking coefficient is proposed. Furthermore, the authors list the factors that have a significant influence on the degree of sieve holes blocking, i.e. the shape of the screened material, the toss indicator and the content of hard-to-screen grains in the feed. This paper is the first one to have thoroughly described the issue of screen blocking.
\end{abstract}

\footnotetext{
Lawinska Katarzyna

k.lawinska@ips.lodz.pl

1 Faculty of Process and Environmental Engineering, Department of Process Equipment, Lodz University of Technology, Wolczanska 175, 90-924 Lodz, Poland

2 Institute of Leather Industry Lodz, Zgierska 73, 91-462 Lodz, Poland
}

Keywords Screening $\cdot$ Sieve $\cdot$ Granular material $\cdot$ Screen blocking

\section{Introduction}

A grain is an element of a permanent disintegrated medium, restricted by an enclosed surface of any shape. Screening is a process that particulate mixtures are often subjected to. The main aim of screening as a method for size classification is to separate a group of grains, whose dimensions fall within the specified limits, from the given material [1]. As a result of the screening process the particulate material is divided into undersized and oversized products [2]. Screening is a random process that takes place as a result of the effect of gravitational forces, which is why its intensity is usually insufficient. In order to accelerate screening in the building aggregates industry, sieves are either flushed with a water jet or purged with an air jet [1]. A factor that inhibits screening is the blocking of sieve holes by the so-called hard-to-screen grains. Those holes that are excluded from the active surface area of the sieve do not participate in the screening process. Furthermore, grains protruding from the surface of the sieve inhibit the motion of the remaining grains and increase the degradation rate of the sieve surface.

There is a large number of publications describing this process and the characteristics of screening machines [3,4]. There are many models describing the screening process. One of them is a model with a discharge function that presents the dependence between the thickness of the material layer on the sieve and the sieve length or screening time [2]. The well-know discrete element method (DEM) involving the integration of ordinary differential equations describing the motion of a free arrangement of material solids in the Cartesian space was used by Li et al. in his paper, treating the sieve 
as an immobile sieve [5]. The authors of paper [6] perform a direct quantitative comparison, across a range of operating conditions, between laboratory scale experiments and simulations using the discrete element method (DEM). It is also important to know the proper method for designing sieves that often involves the method for determining a sieve size, i.e. determining the surface area necessary for performing the planned screening process. Well-known references approach this issue using the probability theory $[7,8]$.

Despite the fact that screening takes place in almost all branches of industry, the issue of sieve holes blocking is not very popular. It was first mentioned in papers by Rendell [9] and Rose [10], however, they did not provide a mathematical description of this unfavourable phenomenon. It was reported by Feller [11] that to evaluate screen performance, both partial passage and clogging of the screen should be considered. A screen rate function, defined as the sum of the passage and clogging rate factors versus relative particle size, was developed to characterize screen performance. It is independent of screening duration and is not limited to a particular size distribution of the material or to one screening duration. It must be noted that sieve holes blocking is not limited to the screening of fine-particulate materials. The issue of sieve holes blocking is not the only problem of this kind encountered in engineering processes used in broadly defined minerals processing. In all processes of filtrating, mixtures containing postmanufacturing contaminated water, originating from water and sludge circuits, there is also blocking of holes in the filtration mesh and the blocking of the filtration deposit pores [12]. Sieve holes blocking is to a large degree a random process that is difficult to describe. No test results nor dependences describing screen blocking have been found in references. During the screening of granular materials in industrial conditions there is often significant blocking of sieve holes; sometimes even more than half of them are blocked. The process of sieve holes blocking is an unfavourable phenomenon, as it reduces the surface area of the lower size fraction flow through the analysed screen. Such a significant reduction of the active surface of a sieve cannot be disregarded when designing and selecting a proper screen. If we are able to estimate the screen blocking coefficient value (for particular screening conditions), i.e. if we are able to estimate the fraction of sieve holes that will be blocked, then it would be possible to determine how large the sieve should be in order to obtain the screening surface area assumed for the process. The authors of this paper have been dealing with this subject for many years $[13,14]$.

\subsection{Shape and size of grains}

Grains properties may be divided into chemical, energyrelated and physical ones. The latter are the ones that to the greatest degree determine the probability of sieve holes blocking and they include grain shape, grain surface roughness, susceptibility to abrasion, hardness [15]. The shape of a particulate material may be determined using a descriptive or mathematical method, while the size may be determined with a screen or sedimentation analysis. Three model shapes of particulate materials are known: roundlike particles (spherical particles), particles with sharp edges (sharp-edged particles) and particles of an irregular shape (irregular particles). Particulate materials that are used in industry may be divided between the model groups according to their shape (Fig. 1).

The grain relative size factor is the fundamental parameter conditioning the screening process and the motion of the material layer along the sieve. It is a dimensionless size that determines the flow of grains through the sieve holes. It is defined as the ratio of the average grain size (diameter) to the size of a sieve hole (Eq. 1). Screening is more difficult when the grain relative size factor is closer to unity.

$\varphi=\frac{\bar{d}}{l}$

Hard-to-screen grains contained in the feed significantly inhibit the screening process and reduce its efficiency. The size of those grains is similar to the size of the sieve holes and they are the ones blocking those holes. Hard-to-screen grains have the following dimensions:

$0.8 l \leq \bar{d} \leq 1.2 l$

Hard-to-screen grains may be divided into undersized grains and blocking grains. Undersized grains are the ones whose dimensions are smaller than or equal to the size of the sieve hole, and that take the most time to pass through the sieve holes. Blocking grains are the ones whose dimensions are equal to or slightly greater than the size of the sieve hole. Those grains do not pass through the sieve holes, remain over the sieve and may clog (block) the sieve holes, thus reducing the screen clearance coefficient.

\subsection{Toss indicator}

The toss indicator has a major effect on the screening efficiency and, consequently, on the value of the sieve holes blocking coefficient. A proper selection of this parameter is of great importance to the process of screening.

$K=\frac{4 \pi^{2} n^{2} A \sin \beta}{g \cdot \cos \alpha}$

where $\mathrm{n}$ - sieve vibration frequency, A-vibration amplitude, $\beta$ - angle of vibrations direction inclination to the sieve surface, $\alpha$-angle of the sieve inclination to the horizontal 

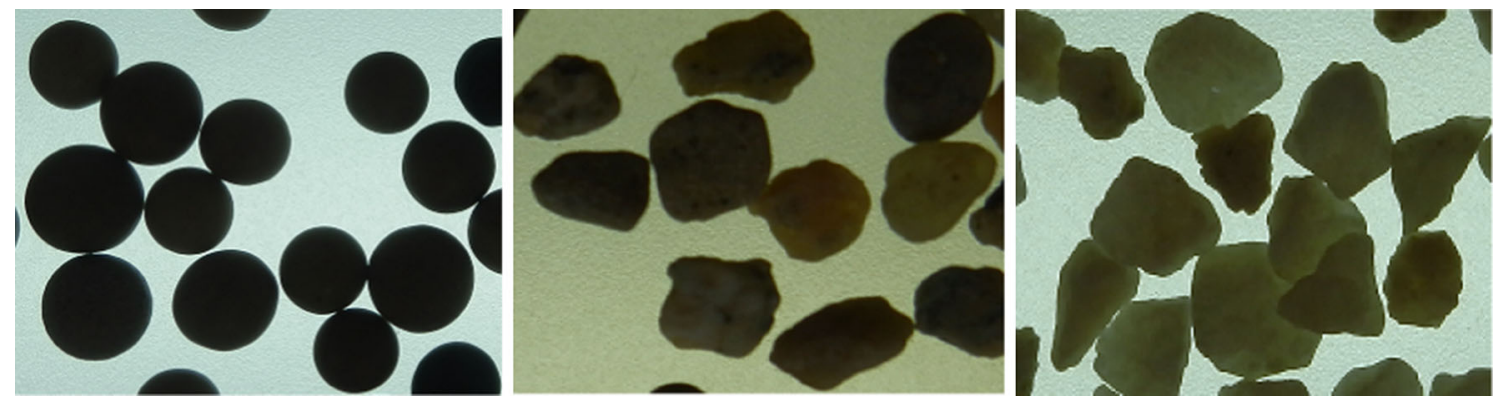

Fig. 1 Model shape of particulate material: spherical grains (agalite), irregular grains (quartz sand), sharp-edged grains (aggregate)

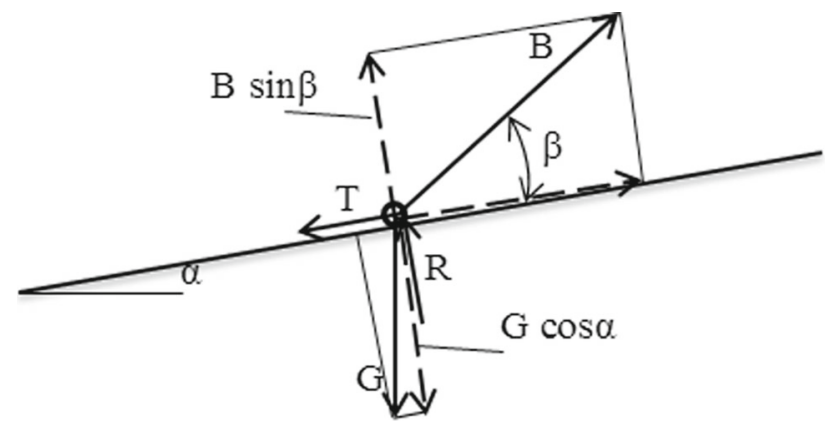

Fig. 2 Forces affecting a grain during a toss: $B$ inertial force, $G$ gravity force, $T$ friction force, $R$ ground reactive force

line. Value $\mathrm{K}$ is a ratio of the normal screen acceleration amplitude component to the normal gravitational acceleration component (Fig. 2) and it is referred to as the toss indicator.

General guidelines concerning proper values of the toss indicator are given in Table 1.

\section{Testing method and materials}

The tests regarding sieve holes blocking were conducted at the Department of Process Equipment of the Lodz University of Technology using two test stations. The screening process included both intermittent (using a laboratory vibrator and control sieves), as well as continuous screening (using a single-plane, circulating screen). The tests involved the empirical determination of the blocking coefficient defined as the ratio of free holes to the total number of sieve holes (Eq. 4).

$f=\frac{n_{\text {free }}}{n_{\text {total }}}$

Particulate materials of three model grain shapes were used for the tests: quartz sand (irregular grains), agalite (spherical grains) and aggregate (sharp-edged grains). Because of the varying shapes of the particulate material the tests were conducted separately for each group. First, each of the material groups was divided using preliminary sieves into fractions within the range of $0.2-2.5 \mathrm{~mm}$. As a result of the preliminary tests, specific fractions of a given material were obtained which enabled the determination of a quantitative share of individual fractions in each material. The mixtures were selected in such a manner that enabled the most significant consideration of the influence of their composition on the sieve holes blocking phenomenon and ensured that they were as close as possible to those that occur in an actual deposit. Materials intended for the tests were used only when dry and free from contamination. The content of hard-to-screen grains $\mathrm{x}_{\mathrm{h}}$ from the range of $\langle 0-100 \%\rangle$ was specified for each mixture.

\subsection{Intermittent tests}

The main part of the tests was performed in a laboratory scale using a vibrator and control sieves on precise woven meshes
Table 1 Ranges of the toss indicator used when classifying particulate materials

\begin{tabular}{ll}
\hline Toss indicator & Application guidelines \\
\hline $\mathrm{K}=2.0-3.3$ & $\begin{array}{c}\text { To be used for classifying easy-to-screen grains that do not show a } \\
\text { tendency for clogging the sieve holes }\end{array}$ \\
$\mathrm{K}=4.5-6.5$ & $\begin{array}{l}\text { To be used when screening average grains (a slightly moist } \\
\text { material with average screen-adhesion properties and minor } \\
\text { tendencies for blocking the sieve holes) } \\
\text { To be used with very hard-to-screen grains, containing much } \\
\text { moisture, easily clogging the sieve holes, with good } \\
\text { screen-adhesion properties }\end{array}$ \\
&
\end{tabular}



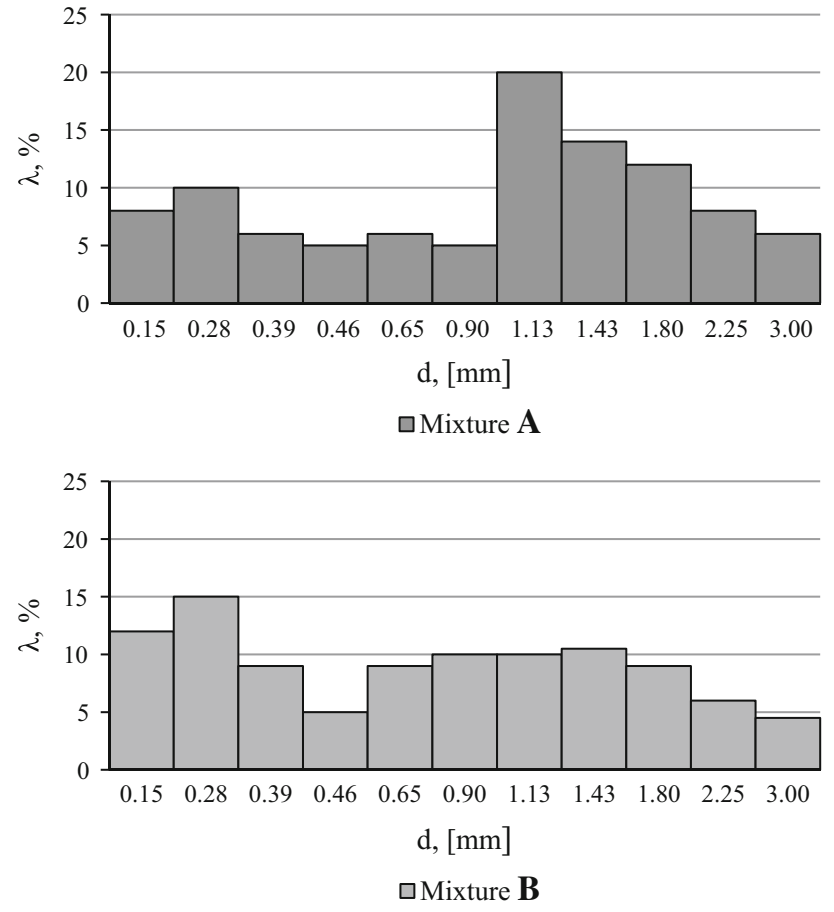

Fig. 3 A particulate composition in the form of a histogram for mixtures $\mathrm{A}$ and $\mathrm{B} ; \lambda$-content of individual fractions of grains, $\mathrm{d}$ - average grain size in a fraction

by Haver\&Boecker (ISO3310-1, ISO3310-2, ASTM-E11). Linear vibrations and flexural vibrations, as well as the regulation of the toss indicator are characteristic of a laboratory vibrator. The main part of the tests involved the screening of each mixture, one by one, through the tested sieve. In total, over 100 mixtures varying in their grain-size composition were tested. $1 \mathrm{~kg}$ of the tested mixture was screened each time.

Examples of grain-size compositions of the mixtures of grains used for the tests are shown in Fig. 3.

A control sieve with the mixture was placed in the vibrator. Prior to the start-up of the vibrator the blocking coefficient $\mathrm{f}_{0}$ (for time $t=0$ ) was calculated in relation to the given particulate material at the moment of it being fed onto the screen. After the vibrator was started, the material was screened through the sieve in time t. After the mixture was screened, the number of free sieve holes was counted at four spots on the screen. Blocked sieve holes are the ones through which grains will not fall out by themselves after the sieve is turned upside down. A template with cut-out frames, each covering 100 sieve holes, was used (Fig. 4). The blocking coefficient was calculated using Eq. 4. The values of coefficient $f$ obtained from four different spots on the screen were averaged and treated as the blocking coefficient for the given sieve in the given time. Blocked grains were removed from the holes and returned to the tested mixture. Screening continued until steady state $t_{\infty}$ was reached (the number of blocked holes in

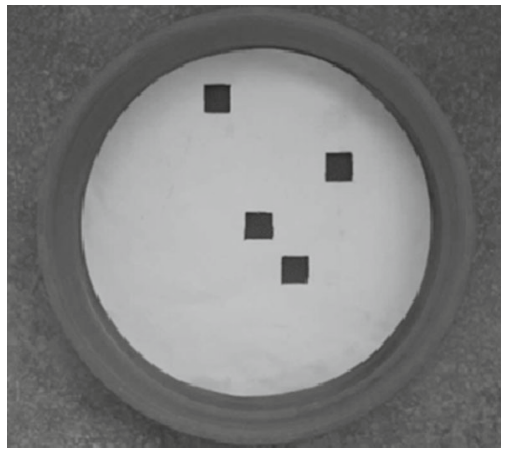

Fig. 4 Control sieve with a template used for calculating the screen blocking coefficient

the sieve is constant, $\left.f_{\infty}\right)$. Such a time span was selected to ensure that the measurement was as precise as possible and that the different stages of the vibrator start-up did not affect the test. After the given screening time elapsed $(1,2,4,8$, $16,32,64,128,256,512,1024,2048 \mathrm{~s})$, the vibrator was turned off. The tests were conducted for five sieves whose hole size was $1=0.5,0.63,0.8,1.0,1.2 \mathrm{~mm}$, and for the toss indicator of $\mathrm{K}=0.62,1.5,1.98,3.5$ and 4.9.

\subsection{Tests of sieve holes blocking on an industrial screen}

For continuous tests a single-plane circulating screen was used that is intended for screening fine and very fine particulate materials. The riddle of this device performs a vibrating motion along the longitudinal plane of the machine, i.e. the plane perpendicular to the sieve surface and parallel to the riddle side walls and to the resultant direction of material on the sieve. The capacity of measurements was $0.08 \mathrm{~kg} / \mathrm{s}$. A single-plane screen has a rectangular, sprung mounted riddle (Fig. 6). A series of tests were conducted for the riddle inclination angle to the horizontal surface of $15^{\circ}$. This is a typical sieve inclination angle used in industrial machines. The toss indicator for this screen is $\mathrm{K}=1.5$. A woven sieve whose square hole dimension is $1=0.63 \mathrm{~mm}$ was used for the measurements. An electric vibrator whose rated speed is 1400 RPM was used for the screen drive. The screen blocking coefficient was measured at 3 spots on the sieve for the time $\mathrm{t}=5,10$ and $30 \mathrm{~s}$ (because of structural, technical reasons) during the continuous operation of the screen. The blocking coefficient was also measured for the time $t=t_{0}$ (at the moment the material is fed onto an immobile sieve). The screening time $\mathrm{t}=30 \mathrm{~s}$ is not the process dynamic equilibrium time, however, in industrial conditions the time of contact between the material and the sieve is not longer than that. In order to determine the screen blocking coefficient, a template with three "cut-outs" was prepared, 100 holes each, in which the number of free holes was counted (Fig. 5). For each time span the test was conducted four times. The 

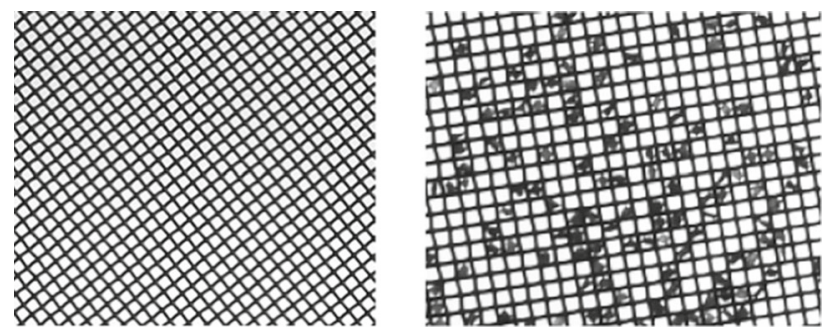

Fig. 5 Mesh of a woven sieve with free and blocked holes

results were averaged for the given measurement time, and the screen blocking coefficient was calculated using Eq. 4 .

\section{Tests results and discussion}

The authors of this paper will present a mathematical model of screen blocking, the effect of the main factors on the value and course in time of the screen blocking coefficient, as well as the method for determining the value of the average coefficient $\mathrm{f}$ for three model shapes of particulate materials. These tests have been conducted by the Division of Granular Material Classification of the Lodz University of Technology for many years.

\subsection{Mathematical model of sieve holes blocking}

Many conventional methods and techniques of mathematical modelling have limited applicability as regards arrangements of particulate materials, and the obtained models have special features and restrictions. The application of fundamental principles of science, however, is preferred [16].

The screen blocking phenomenon is a result of two simultaneous phenomena: pure blocking and pure unblocking of sieve holes. The resultant rate of the process will therefore equal the sum of both elementary rates. The main difficulty in the screening process modelling is the fact that the screened material is a collection of various grains. A mathematical description of the screening process is only possible if we assume as certain model that is close to reality. For the mathematical model of the blocking process it was assumed that $\mathrm{H} / \mathrm{D}_{\mathrm{z}}<0.1$, which conditions the free discharge of the material through the sieve bottom. His the height (thickness) of the particulate material on the sieve, while $\mathrm{D}_{\mathrm{z}}$ is the sieve diameter. Furthermore, it was assumed that the blocking coefficient is not a function of the thickness of the material layer on the sieve. A constant thickness of material on the sieve was assumed. In this model we assume that the hydrostatic pressure of the material layer does not affect sieve holes blocking and the screen blocking coefficient. The feed is interpreted as a particulate stream fed uniformly on the entire surface of the sieve, in which the particulate material is evenly dis- tributed. The consequence of a non-uniform feeding of the feed onto the entire surface of the sieve is the fact that not the entire surface of the sieve is utilised. This results in an inefficient screening process. At the moment of the material being fed onto the sieve $t=0$ the blocking coefficient equals $f=f_{0}$. This means that the screen has not performed a vibration yet, however, there are already some blocked sieve holes. With time $t=t_{\infty}$ the dynamic equilibrium of sieve holes unblocking and blocking processes stabilizes. Then the blocking coefficient $\mathrm{f}=\mathrm{f}_{\infty}$ and its value cease to change. With the above-mentioned assumptions it may be concluded that the rate of sieve holes blocking is proportional to the number of free holes. The more free holes, the greater the tendency for their clogging. Mathematically it can be formulated as follows:

$u_{1}=-\frac{d f}{d t}=k_{1} \cdot f$

$\mathrm{k}_{1}$ is the screen blocking constant. By integrating and rearranging Eq. (5) we obtain

$f=f_{0} \cdot e^{-k_{1} \cdot t}$

Furthermore, it may be assumed that the greater the blocked surface fraction, $(1-f)$ the higher the tendency for sieve holes unblocking. Similarly to the case of Eq. (5) we may write

$u_{2}=-\frac{d f}{d t}=-k_{2} \cdot(1-f)$

After transformations we obtain:

$f=1-\left(1-f_{0}\right) \cdot e^{-k_{2} \cdot t}$

$\mathrm{k}_{2}$ is the screen unblocking coefficient. As the processes of screen blocking and unblocking oppose each other, their rates have a reverse sign. Assuming that $\mathrm{f}_{0}>\mathrm{f}_{\infty}, \mathrm{u}_{1}>\mathrm{u}_{2}$ and the resultant rate $\mathrm{u}=\mathrm{u}_{1}+\mathrm{u}_{2}$ will have the same sign as the blocking rate $\mathrm{u}_{1}$. We will obtain the following mathematical formula:

$u=u_{1}+u_{2}$

$-\frac{d f}{d t}=k_{1} \cdot f-k_{2} \cdot(1-f)$

Boundary conditions for $\mathrm{t}=0, \mathrm{f}=\mathrm{f}_{0}$, while for $\mathrm{t}=\mathrm{t}_{\infty}, \mathrm{f}=$ $\mathrm{f}_{\infty}$ also show that the following dependence must be fulfilled:

$f_{\infty}=\frac{k_{2}}{k_{1}+k_{2}}$

Considering dependence (11) and transforming Eq. (10) we obtain

$f-f_{\infty}=\left(f_{0}-f_{\infty}\right) e^{-\left(k_{1}+k_{2}\right) \cdot t}$ 

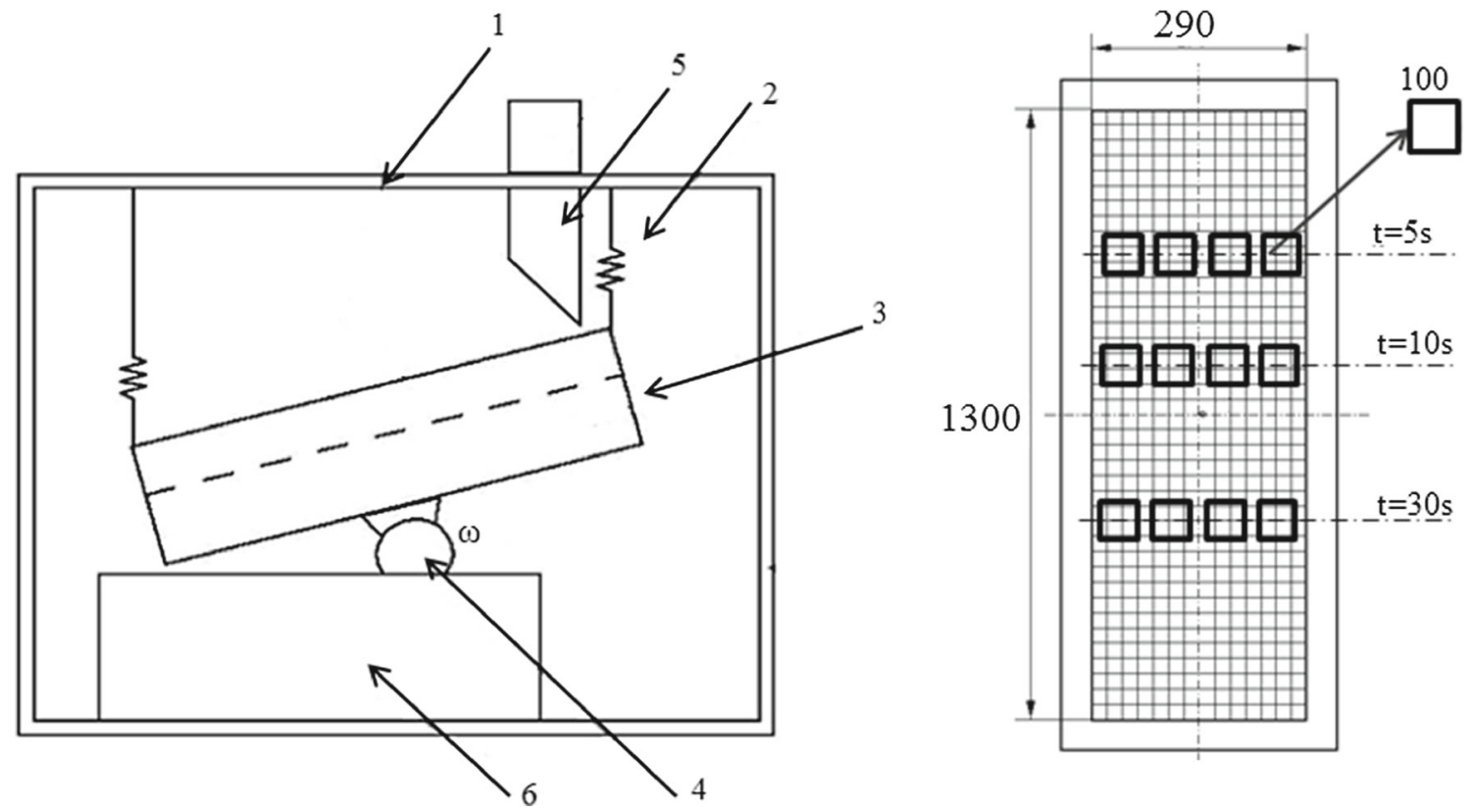

Fig. 6 Test station with a single-plane screen: 1 load-bearing structure, 2 screen suspension including springs and chains, 3 riddle with sieve, 4 electric vibrator, 5 charging funnel with a sliding gate, 6 container for the

Considering that $k=k_{1}+k_{2}$ we obtain dependence (12) that is the fundamental equation for determining the screen blocking coefficient in time $t$.

$f=f_{\infty}+\left(f_{0}-f_{\infty}\right) e^{-k \cdot t}$

The constants of this equation are expressed with formulas (Eqs. 14, 15) and are determined for the given material and sieve arrangement.

$$
\begin{aligned}
f_{\infty} & =\frac{f_{0} \cdot f_{n+1}-f_{n}^{2}}{f_{0}-2 \cdot f_{n}+f_{n+1}} \\
k & =\frac{1}{t_{n}} \cdot \ln \left|\frac{f_{0}-f_{\infty}}{f_{n}-f_{\infty}}\right|
\end{aligned}
$$

The modelling of sieve holes blocking was based on an exponential mathematical model $[17,18]$. In order to asses the correctness of the screen blocking mathematical model, the figure of merit for the model fitting the empirical data $R^{2}$ was also calculated. In most of the modelled arrangements, the value of coefficient $R^{2}$ exceeded 0.9 prove that the empirical data satisfactorily fit the mathematical model. The discrepancy between the mathematical modelling results and the empirical data may result from the fact that the mathematical model does not account for such parameters as, for example, the shape of the sieve hole or the sieve type. If we have decided to account for such parameters, the mathematical model would become much more extensive and complex. The correctness of the mathematical model of screen blocking is confirmed for two characteristic courses of the screen block- screened material and the single-plane screen sieve with marked measurement points

ing coefficient dependence in the function of time (Figs. 7, 8; Table 2).

\subsection{Main factors affecting sieve holes blocking}

Factors greatly affecting sieve holes blocking in the tested arrangement include: the shape of the screened material, the content of hard-to-screen grains in the feed and the toss indicator of the screening machine. The determined (nonvariable) value of the screen blocking coefficient results from the sieve holes blocking and unblocking dynamic equilibrium. Data labels for the values of $f_{0}$ and $f_{\infty}$ have been added in the diagrams below. The conducted tests show that the sharp-edged material blocks the sieve holes to the greatest degree (Fig. 9). For these cases the value of the screen blocking coefficient $f$ is the lowest. The most free holes occur when screening spherical materials - that is when the screen blocking process is the least significant. A different course of the screen blocking coefficient dependence can be observed for an irregular shape of grains of the tested mixture (for sand). In this case the value of coefficient $f$ increases with the duration of the screening process (so the number of blocked holes decreases). It may be concluded that among various grain shapes it is easier for irregular grains to fall out of sieve holes as a result of vibrating devices vibrations. The hight content of hard-to-screen grains in the feed greatly inhibits the screening process. In most cases the number of blocked sieve holes increases with an increase in the content of hardto-screen grains (Fig. 10). The machine toss indicator has a significant influence on the screen blocking phenomenon. 

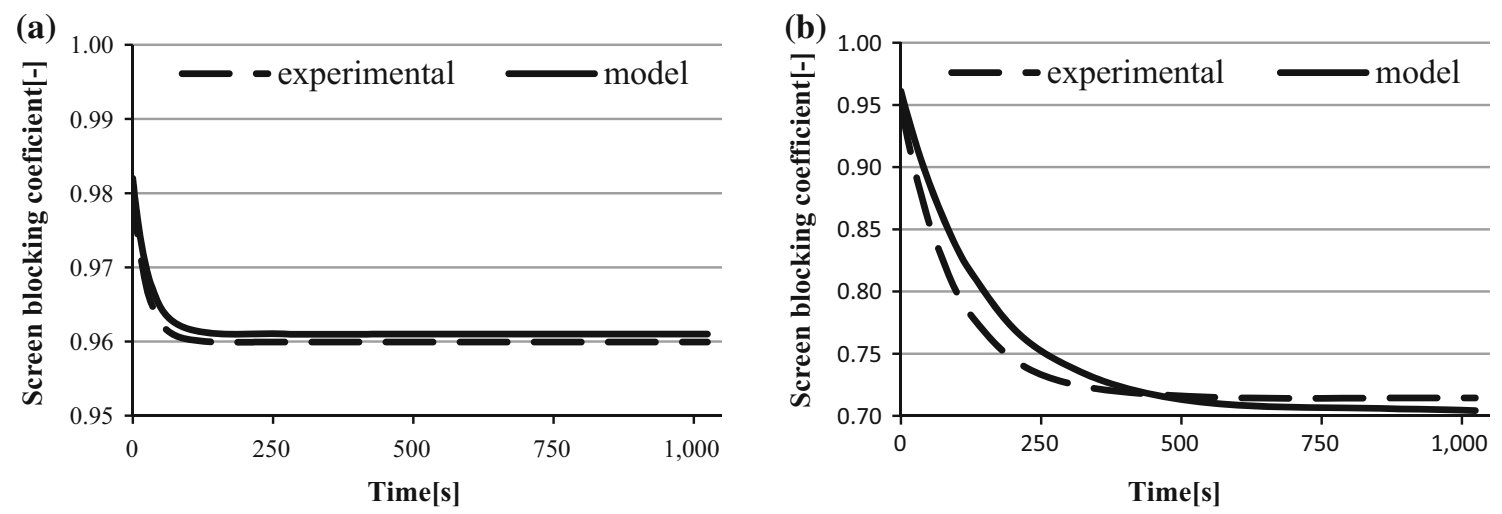

Fig. 7 Model and theoretical values of the screen blocking coefficient for $\mathrm{f}_{0}>\mathrm{f}_{\infty}$ : $\mathbf{a} \mathrm{K}=1.5$; $\mathbf{b} \mathrm{K}=3.5$ (spherical grains, sieve $1=1 \mathrm{~mm}$ )

(a)

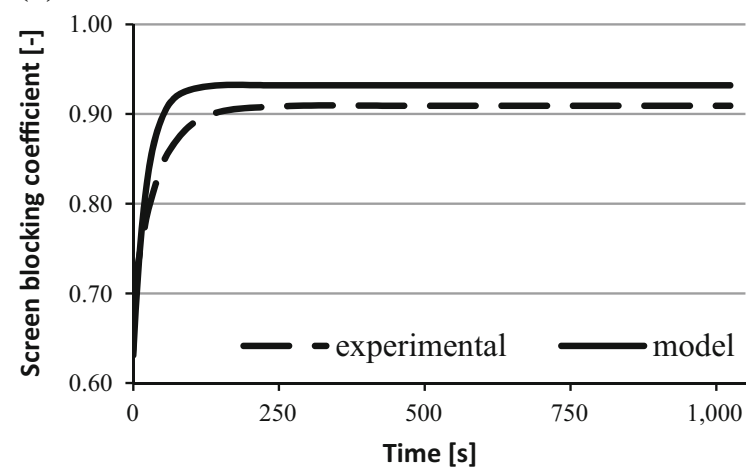

(b)

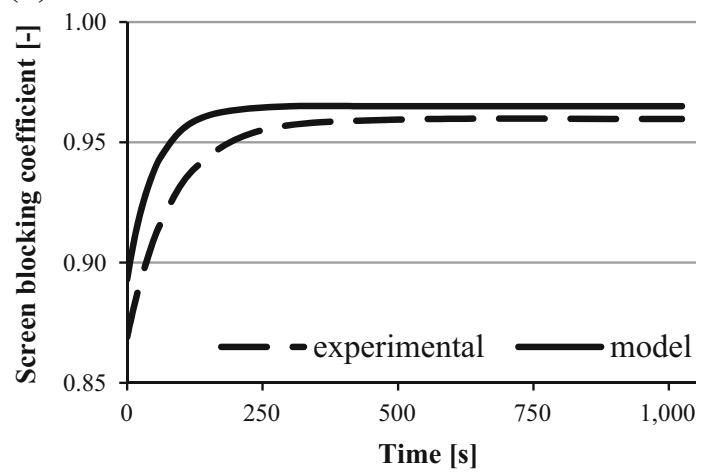

Fig. 8 Model and theoretical values of the screen blocking coefficient for $f_{0}<f_{\infty}$ : a $K=1.5 ; \mathbf{b} K=3,5$ (irregular grains, sieve $1=0.5 \mathrm{~mm}$ )

Table 2 Model and empirical values of the screen blocking coefficient in the function of time for the presented arrangements (Figs. 7, 8)

\begin{tabular}{lll}
\hline Figures & Data & $f=f_{\infty}+\left(f_{0}-f_{\infty}\right) \cdot e^{-k \cdot t}$ \\
\hline $7 \mathrm{a}$ & Experimental & $f=0.960+0.0209 \cdot e^{-0.0421 \cdot t}$ \\
& Model & $f=0.961+0.0210 \cdot e^{-0.0353 \cdot t}$ \\
$7 \mathrm{~b}$ & Experimental & $f=0.714+0.235 \cdot e^{-0.0101 \cdot t}$ \\
& Model & $f=0.704+0.257 \cdot e^{-0.00667 \cdot t}$ \\
8a & Experimental & $f=0.909-0.214 \cdot e^{-0.0234 \cdot t}$ \\
& Model & $f=0.932-0.301 \cdot e^{-0.0433 \cdot t}$ \\
8b & Experimental & $f=0.960-0.0907 \cdot e^{-0.0119 \cdot t}$ \\
& Model & $f=0.965-0.072 \cdot e^{-0.0197 \cdot t}$ \\
\hline
\end{tabular}

Higher values of the toss indicator $\mathrm{K}$ reduce the number of blocked sieve holes, and the value of the coefficient $\mathrm{f}$ pursues unity. Furthermore, the value of the toss indicator also affects the character (monotonicity) of the dependence of coefficient $\mathrm{f}$ in the function of time (Fig. 11).

From the results of tests of the screen blocking coefficient conducted during intermittent screening (laboratory vibrator and control sieves), as well as continuous screening (single-plane screen), the ones with the most similar grainsize composition of the tested mixtures, the model shape

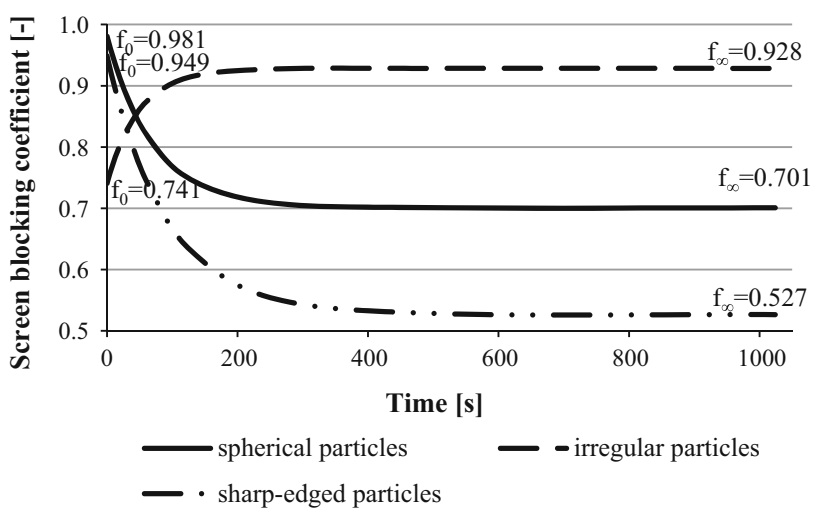

Fig. 9 Screen blocking coefficient for three model shapes of particulate materials $\left(\mathrm{K}=1.5, \mathrm{x}_{\mathrm{h}}=90 \%\right.$, sieve $\left.1=1 \mathrm{~mm}\right)$

of their grains and screening parameters $(K=1.5)$ were selected for comparison.

The comparison of results obtained from intermittent and continuous screening (Fig. 12) proves that the conclusions regarding the screen blocking coefficient drawn from the small-scale tests (using laboratory vibrators and control sieves for the purpose of covering the widest possible scope of parameters variability) could be used in industrial applications (in actual process conditions). 


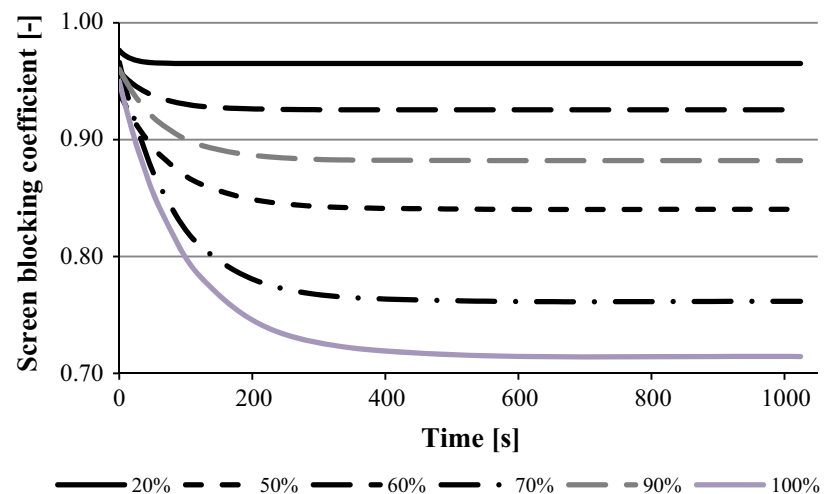

Fig. 10 Screen blocking coefficient for mixtures of varying hard-toscreen grains content $\mathrm{x}_{\mathrm{h}}(\mathrm{K}=1.98$, spherical grains, sieve $\mathrm{l}=0.5 \mathrm{~mm})$

\section{Method for determining the average screen blocking coefficient}

The possibility of estimating the value of the screen blocking coefficient is an important piece of information on sieve holes blocking. This information may be used for designing

(a)

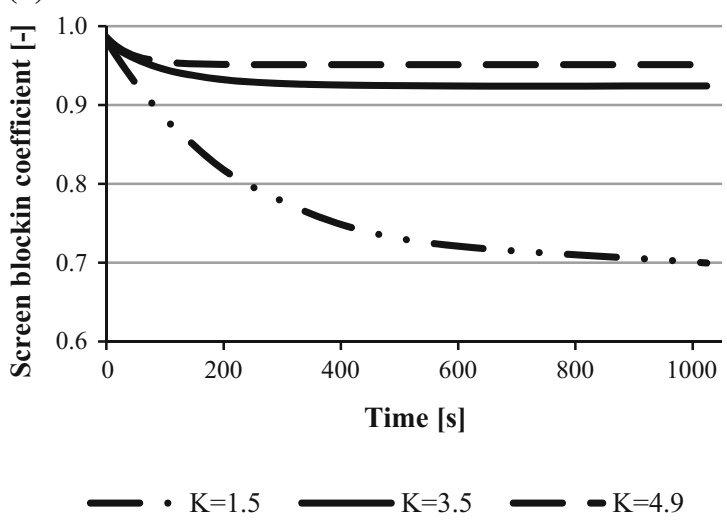

and optimising an industrial screening process. In order to determine the screen blocking coefficient its constant value is necessary.

In real-life conditions the dependence of the blocking coefficient in time often runs along the curve shown in Fig. 13.

Reaching the state of process equilibrium for time $t_{\infty}$ is much longer than the duration of the transfer process (the statistical time the material remains on the sieve). The time the material remains on an industrial sieve $\left(t_{p}\right)$ is usually between several and $60 \mathrm{~s}$. Considering this, it is necessary to determine the average value of the screen blocking coefficient in the tested arrangements.

On the basis of the functional courses of the blocking coefficient (obtained empirically) for each material and sieve arrangement, the value of the arithmetic mean from the range of $\left\langle\mathrm{f}_{0}, \mathrm{f}_{\infty}\right\rangle$ was determined. An average value of the screen blocking coefficient $\mathrm{f}^{*}$ was obtained for each of the tested arrangements. Considering the conclusions from the previous chapter (Sect. 3.2) a method for determining the average screen blocking coefficient for fine-particulate materials in

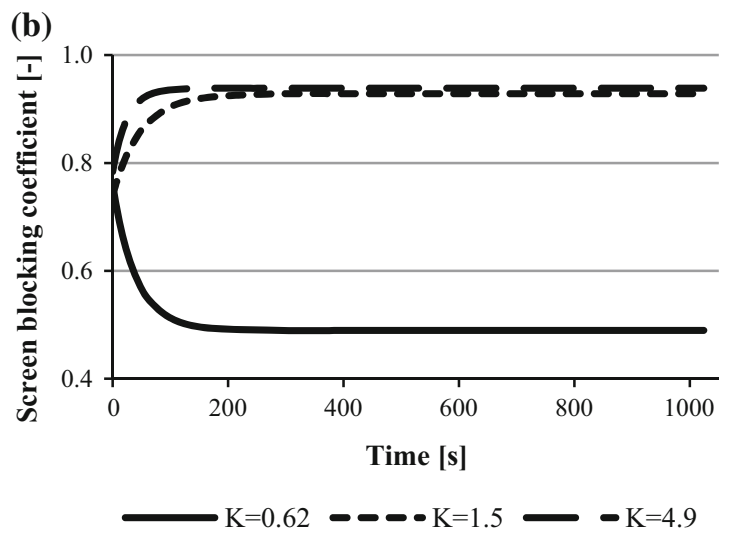

Fig. 11 Screen blocking coefficient for varying values of the toss indicator: a (spherical grains, $x_{h}=65 \%$, sieve $1=0.5 \mathrm{~mm}$ ); $\mathbf{b}$ (irregular grains, $\mathrm{x}_{\mathrm{h}}=75 \%$, sieve $1=0.5 \mathrm{~mm}$ )

(a)

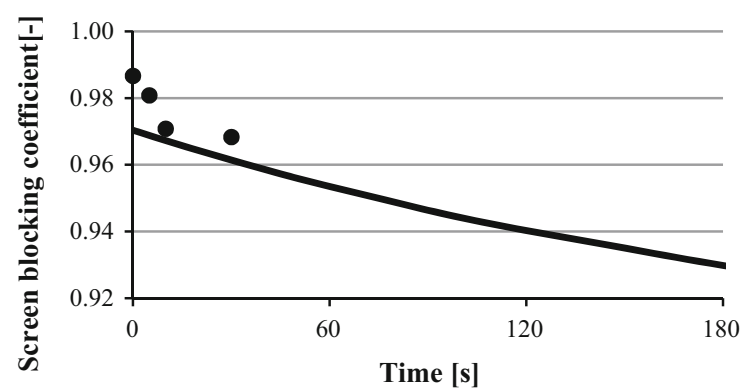

intermittent screening

- continuous screening (b)

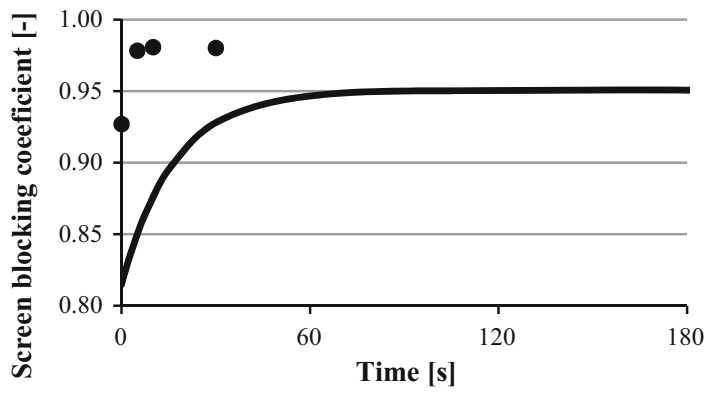

- intermittent screening continuous screening

Fig. 12 Screen blocking coefficient for mixtures for intermittent and continuous screening: $\mathbf{a} \mathrm{f}_{0}>\mathrm{f}_{\infty}$ (irregular grains, $\mathrm{K}=1.5$, $\mathrm{x}_{\mathrm{h}}=50 \%$, sieve $1=0.63 \mathrm{~mm}$ ); $\mathbf{b} \mathrm{f}_{0}<\mathrm{f}_{\infty}$ (spherical grains, $\mathrm{K}=1.5, \mathrm{x}_{\mathrm{h}}=50 \%$, sieve $\mathrm{l}=0.63 \mathrm{~mm}$ ) 


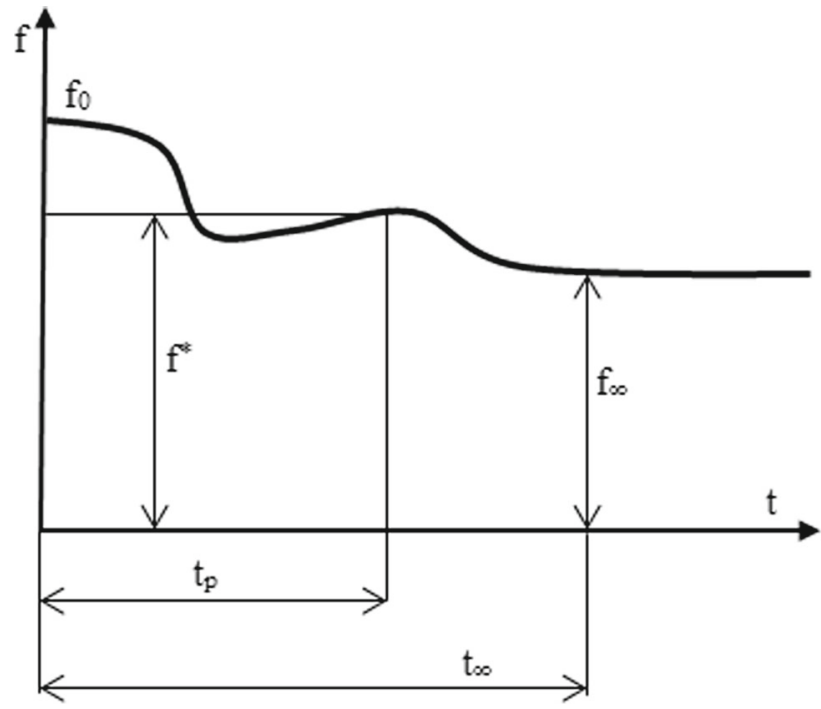

Fig. 13 The actual course of the screen blocking coefficient in time: $\mathrm{f}_{0}$ - blocking coefficient at the moment the material is fed onto the sieve, $\mathrm{f}_{\infty}$-blocking coefficient for the steady state, $t_{\mathrm{p}}$-industrial process duration

the function of the main factors affecting its value, i.e. the toss indicator and the content of hard-to-screen grains in the feed was specified. The quantitative description of the blocking phenomenon was presented for each model shape of the screened material separately. In order to find the functional dependence of the average blocking coefficient on the toss indicator and the content of hard-to-screen grains, the functions of Mthcad13 were used. For the determination of the value of an average screen blocking coefficient, a model of function of multiple variables that is the $\mathrm{n}$ degree polynomial was proposed:

$y\left(x_{1}, x_{2}, \ldots, x_{n}\right)=\sum_{i=1}^{i=n} a_{i} \cdot x_{1}^{P_{i, 1}} \cdot x_{2}^{P_{i, 2}} \cdot x_{n}^{P_{i, n}}$

This method is described in [19], and with reference to the sieve blocking coefficient it is described in [20]. When defining the algorithm for determining the power matrix and the value of coefficients, a model of an n-degree polynomial of a function of two variables $\left(\mathrm{K}, \mathrm{x}_{\mathrm{h}}\right)$ was studied. The obtained forms of a third degree polynomial of the function of two variables and the values of coefficients $R^{2}$ with a division into model shapes of the particulate material are listed in table below. The values of the determination coefficient $\mathrm{R}^{2}$ show that the model is very well fitted to the results of experiments.

On the basis of Table 3, one can calculate the value of an average screen blocking coefficient and determine the active surface area of the screen, as well as the clearance coefficient, at the stage of planning the screening process and screening machine.

$F_{e f}=f^{*} \cdot F \cdot A_{0}$

where $\mathrm{F}$ is the screen surface area. The sieve clearance coefficient, $A_{0}$, defined as the ratio of the holes surface area to the total screen surface area, provides information on the screening capacity of a particular screen.

$p=\frac{F_{e f}}{F}$

\section{Conclusions}

Sieve holes blocking is a complex and random phenomenon. This paper provides a mathematical description of the sieve holes blocking process, and the obtained analytical expressions provide for the determination of characteristic values of the process. The proposed exponential mathematical model describes the blocking process well.

The proposed method for determining the screen blocking coefficient in the function of the main factors affecting its values is the first written proposal for defining the process of sieve holes blocking. The value and the course in time of the blocking coefficient is a very important parameter of the screening process. Determination of the theoretical dependences that are used for its specification
Table 3 A list of coefficients and powers of degree polynomial model of an average screen blocking coefficient $\mathrm{f}^{*}$ in the function of the toss indicator $\mathrm{K}$ and the content of third hard-to-screen grains $\mathrm{x}_{\mathrm{h}}$

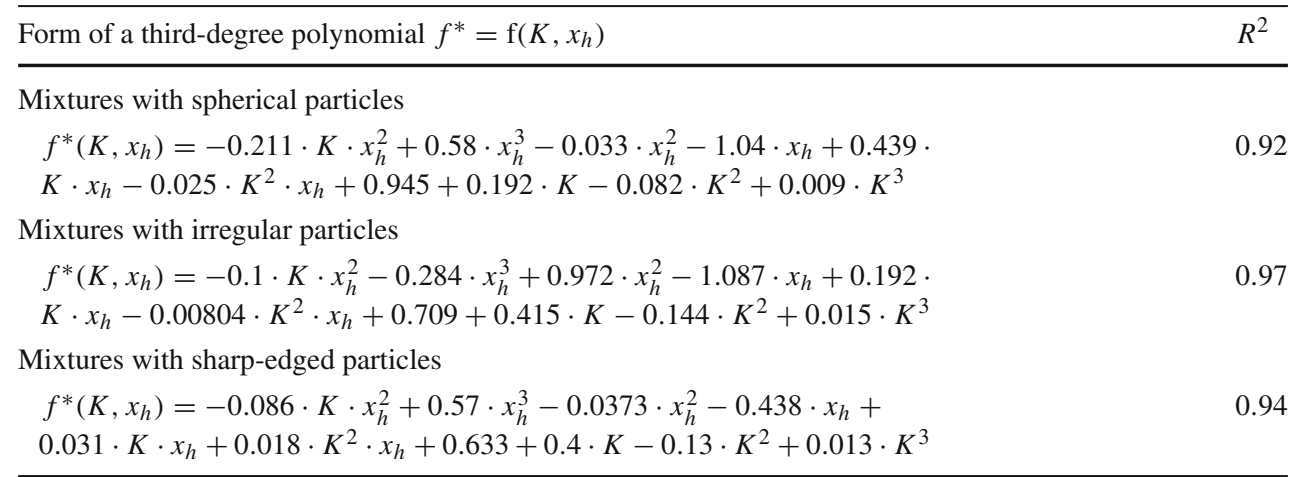


enable a major increase in the efficiency of the screening process. The obtained and published tests results regarding sieve holes blocking provide important information to all users of screens. Conclusions from the research are addressed to a large group of people working on screening processes and provide guidelines for working in actual process conditions, above all, in mining, metallurgy, coal processing, civil engineering, environmental protection, as well as in food, chemical, pharmaceutical and leather industries. The scale of the screening is so large because millions of tons of products are being screened every single day.

Acknowledgments The research work has been carried out within project: "The phenomenon of sieve holes blocking during the screening of fine-grained materials" financed by Faculty of Process and Environmental Engineering, Lodz University of Technology (Grant 2014/501/10-34-2-1044) and this study was performed as a part of chartered assignment W-10/7534/2014 Dz.St.-1.

Open Access This article is distributed under the terms of the Creative Commons Attribution 4.0 International License (http://creativecomm ons.org/licenses/by/4.0/), which permits unrestricted use, distribution, and reproduction in any medium, provided you give appropriate credit to the original author(s) and the source, provide a link to the Creative Commons license, and indicate if changes were made.

\section{References}

1. Feder, S., Keska, W., Wlodarczyk, K.: Pneumatic aiding of the process of screening granular mixtures using a screening table. Agric. Eng. 4(102), 263-270 (2008)

2. Wodzinski, P.: Fine screening and screens. J. Pol. Miner. Eng. Soc. 41-57 (2001)

3. Akhmadiev, F.G., Gizzatov, R.F.: Separation processes of granular materials by sizes at the sieve classifiers. J. Chem. Chem. Eng. 7, 56-63 (2013)

4. Beecmans, J.M., Hu, E., Germain, R., McIntyre, A.: Performance characteristics of a probability screening machine. Powder Technol. 43(3), 249-256 (1985)
5. Li, J., Webb, C., Pandiella, S.S., Campbell, G.M.: Discrete particle motion on sieve-a numerical study using the DEN simulation. Powder Technol. 133, 190-203 (2003)

6. Delaney, G.W., Cleary, P.W., Hilden, M., Morrison, R.D.: Testing the validity of the spherical DEM model in simulating real granular screening processes. Chem. Eng. Sci. 68(1), 215-226 (2012)

7. Bandemer, H., Espig, D.: A mathematical model of the batch sieving procedure. Powder Technol. 20, 227-231 (1987)

8. Jansen, M.L., Glastonbury, J.R.: The size separation of particles by screening. Powder Technol. 1, 334-343 (1967/68)

9. Rendell, M.: Separation of Particles by Sieving and Screening. Ph. D. Thesis, University of London (1964)

10. Rose, H.E.: Mechanism of sieving and screening. Trans. Inst. Min. Metall. Sect. C Miner. Proc. Extr. Metall. 86, 101-114 (1977)

11. Feller, R.: Screening analysis considering both passage and clogging. Trans. ASAE 23(4), 1054-1056 (1980)

12. Piecuch, T., Piekarski, J., Malatanska, G.: The equation describing the filtration process with compressible sediment accumulation on a filter mesh. Arch. Environ. Prot. 39(1), 93-104 (2013)

13. Lawinska, K., Wodzinski, P.: Determination of the effective sieve blocking coefficient. Physicochem. Probl. Miner. Process. 48(1), 247-252 (2012)

14. Lawinska, K., Wodzinki, P., Modrzewski, R.: Verification of the mathematical model of the screen blocking process. Powder Technol. 256, 506-511 (2014)

15. Baic, I.: Analysis of the chemical, physical and energetic parameters of coal sludge deposits inventoried in the Silesian Province. Annu. Set Environ. Prot. 15, 1525-1548 (2013)

16. Tumidajski, T.: Actual tendencies in description and mathematical modeling of mineral processing. Miner. Resour. Manag. 26(3), $111-123(2010)$

17. Blasinski, H., Wodzinski, P.: Sieve holes blocking. Sci. Pap. Lodz Univ. Tech. Chem. Eng. 2(177), 63-78 (1973)

18. Blasinski, H., Wodzinski, P.: Research process of sieve holes blocking. Sci. Pap. Lodz Univ. Tech. Chem. Eng. 6(232), 13-23 (1976)

19. Palaczek, W.: Mathcad 12, 11, 2001i, 2001, 2002 in the Algorithms. Academic Publishing House EXIT, Warsaw (2005)

20. Lawinska, K., Wodzinski, P., Modrzewski, R.: A method for determining sieve holes blocking degree. Physicochem. Probl. Miner. Process. 51(1), 15-22 (2015) 